\title{
ANÁLISE DA COMPOSIÇÃO CORPORAL E PREVALÊNCIA DE DOENÇAS CRÔNICO- DEGENERATIVAS EM ADULTOS DE DIFERENTES FAIXAS ETÁRIAS
}

\author{
Rodrigo Evangelista ${ }^{1}$, Robson Chacon Castoldi ${ }^{2}$, Alan José Barbosa Magalhães ${ }^{1}$, João Henrique Lyrio \\ Machado $^{1}$, Guilherme Akio Tamura Ozaki ${ }^{1}$, Giovana Rampazzo Teixeira ${ }^{1}$; Regina Celi Trindade \\ Camargo $^{1}$, José Carlos Silva Camargo Filho ${ }^{1}$, Olga Cristina de Mello Malheiro ${ }^{1}$. \\ ${ }^{1}$ Universidade Estadual Paulista, Presidente Prudente - SP. Faculdade de Ciências e Tecnologia, UNESP, Presidente \\ Prudente - SP. ${ }^{2}$ Universidade do Oeste Paulista, UNOESTE, Presidente Prudente - SP. E-mail: \\ castoldirc@yahoo.com.br
}

\section{RESUMO}

Percebe-se atualmente que fatores associados ao excesso de peso e obesidade são os maiores problemas de saúde pública em todo o mundo. Além disso, com o avançar da idade, existe uma tendência à propensão de doenças. Nesse sentido, o objetivo do presente estudo foi analisar envelhecimento associado obesidade ou sobrepeso e ocorrência de doenças crônico-degenerativas em indivíduos de diferentes faixas etárias. A amostra foi composta por 77 indivíduos, sendo 40 homens 37 mulheres, com média de idade de 30 anos $( \pm 9,36)$, residentes em Presidente Prudente SP. Foi mensurado o Índice de Massa Corporal (IMC), Percentual de Gordura Corporal (\%GC) e Obesidade Abdominal (OAB). Além disso, foram investigados os casos de Doenças CrônicoDegenerativas (DCD). Foi observado que indivíduos com idade até 30 anos possuem menor prevalência de sobrepeso, obesidade e doenças crônico-degenerativas. Dessa forma, é possível concluir que a ocorrência de excesso de peso e obesidade, bem como o surgimento de complicações patológicas, ocorre com maior frequência em indivíduos com idade mais avançada.

Palavras-chave: Obesidade; Doenças Crônico-Degenerativas; Envelhecimento.

\section{BODY COMPOSITION AND CHRONIC-DISEASE PREVALENCE IN ADULTS WITH DIFFERENT AGE GROUPS}

\begin{abstract}
It is noticed nowadays that factors associated with overweight and obesity are major public health problems worldwide. Furthermore, with advancing age, there is a tendency to disease propensity. In this sense, the objective of this study was to analyze the association of aging with the overweight and the occurrence of chronic diseases in individuals of different age groups. The sample consisted of 77 individuals, 40 men and 37 women, mean age of 29.99 years ( \pm 9.36 ), living in Presidente Prudente - SP. Were measured for body mass index (BMI), Body Fat Percentage (BF\%) and Abdominal Obesity (OAB). In addition, we investigated the cases of Chronic Degenerative Diseases (DCD). It was observed that individuals aged 30 years have a lower prevalence of overweight, obesity and chronic degenerative diseases. Thus, we conclude that the occurrence of overweight and obesity, as well emergence of pathological involvement, the occurrence with more frequency in individuals with higher age.
\end{abstract}

Keywords: Obesity, Chronic Degenerative Diseases, Aging. 


\section{INTRODUÇÃO}

A obesidade tem se tornado atualmente problema de saúde pública mundial. O número de indivíduos com sobrepeso tem aumentado consideravelmente, seja em países desenvolvidos ou em desenvolvimento ${ }^{1}$. Com a crescente mudança de alterações ambientais e comportamentais especialmente nos hábitos alimentares a obesidade mundial está sendo considerada epidemia ${ }^{2}$.

Dados globais indicam que doenças crônicas como Hipertensão Arterial, Diabetes Tipo II, Doenças Osteomusculares, entre outras, são responsáveis por cerca de $59 \%$ dos 56,5 milhões de óbitos anuais em todo o mundo, e por $45,9 \%$ do total de enfermidades $^{1}$. No Brasil a prevalência tais doenças são responsáveis por aproximadamente $66 \%$ dos óbitos e, desse total, $4,4 \%$ são ocasionadas por doenças cardiovasculares. $\mathrm{Na}$ literatura diversos estudos comparando indivíduos com peso normal com indivíduos obesos, assim a obesidade está associada ao maior risco para o desenvolvimento de doenças crônicodegenerativas $(D C D)^{3,4}$. Doenças como Diabetes Mellitus II e Hipertensão Arterial Sistêmica (HAS), favorecem o desenvolvimento de doenças cardiovasculares $(\mathrm{DCV})^{5}$.
A combinação de índice de massa corporal elevado (IMC $\geq 30 \mathrm{Kg} / \mathrm{m}^{2}$ ) está associada à hipertensão arterial atribuída à resistência de insulina principalmente naqueles indivíduos que concentram maior parte de gordura na região abdominal ${ }^{6}$. Alguns índices antropométricos como circunferência da cintura e o IMC são medidas correlacionadas entre si e utilizados para comparar o excesso de peso associado a fatores de risco que podem desenvolver DCV. Nesse sentido, Indivíduos que apresentam medida da circunferência abdominal maior que $88 \mathrm{~cm}$ para mulheres e maior que $102 \mathrm{~cm}$ para homens, caracterizam a distribuição central de gordura e estão mais propensos a desenvolver doença cardiovascular ${ }^{7}$.

O exercício físico é um meio nãofarmacológico no controle de peso e redução da gordura corporal. Essa medida está associada à redução dos riscos de doenças, em especial, as $\mathrm{DCV}^{8}$. Praticantes de atividades físicas, sejam elas aeróbias ou anaeróbias, tendem a diminuir $\mathrm{o}$ peso corporal, fortalecer a musculatura e reduzir o risco de surgimente de $D_{C D}^{9}$.

Nesse sentido, altos índices de gordura corporal podem estar diretamente ligados ao surgimento de patologias e consequentemente, podem prejudicar a vivência e realização de atividades físicas do 
cotidiano. Sendo assim, o objetivo do presente estudo foi analisar a associação da idade com excesso de peso e ocorrência de doenças crônico-degenerativas em indivíduos de diferentes faixas etárias.

\section{MATERIAIS E MÉTODOS}

Amostra

Participaram deste estudo 77 indivíduos, sendo 40 homens 37 mulheres, com média de idade de 29,99 anos $( \pm 9,36)$, frequentadores de uma academia de musculação e residentes na cidade de Presidente Prudente - SP, Brasil. Os indivíduos foram divididos em dois grupos, grupo1 indivíduos com idade até 30 anos e grupo 2 com idade superior a 30 anos. Todos os procedimentos seguiram sob princípios éticos da pesquisa, com aprovação do Comitê de Ética e Pesquisa da Faculdade de Ciências e Tecnologia, FCT-UNESP (Campus Presidente Prudente), protocolo número 43/2010.

Foram analisadas as variáveis antropométricas dos dois grupos. Dessa forma, foram mensurados o Índice de Massa Corporal (IMC); o Percentual de Gordura Corporal $(\% \mathrm{GC})$ e a presença ou ausência de Obesidade Abdominal (OAB), bem como a verificação do histórico de doenças crônicodegenerativas de cada participante.

Variáveis de Estudo - Instrumentos de medida

O peso corporal foi mensurado com a utilização de uma balança digital, com precisão de 0,1kg e a capacidade máxima de $180 \mathrm{~kg}$. A estatura foi aferida com a utilização de um estadiômetro fixo de madeira, com precisão de $0,1 \mathrm{~cm}$ e extensão máxima de dois metros (marca Sanny ${ }^{\circledR}$ ).

Para a variável de circunferência da cintura (CC), os avaliados permaneciam na posição ortostática, respirando normalmente e com os braços descontraídos ao lado do tronco. Todos os registros foram feitos ao final de uma expiração normal. Ambas as medidas de circunferências foram efetuadas com uma fita métrica metálica, com precisão de $0,1 \mathrm{~cm}$ e extensão máxima de $2 \mathrm{~m}$ (marca Sanny $\left.{ }^{\circledR}\right)$.

O percentual de gordura corporal (\%GC) foi obtido por meio de uma balança de bioimpedância da marca Lumina $^{\circledR}$ (modelo Plenna). A resistência e a reatância corporal total foram medidas com o uso de um analisador acoplado ao aparelho na posição vertical.

O cálculo do IMC foi efetuado por meio da divisão do valor do peso corporal $(P)$, em quilogramas, pela estatura (E), em metros, elevada ao quadrado $\left(I M C=P / E^{2}\right)$. A partir dos valores encontrados no IMC, os avaliados foram classificados de acordo com a Organização Mundial de Saúde ${ }^{10}$ (Tabela 1). Dessa maneira, indivíduos que apresentaram valores até $24,9 \mathrm{Kg} / \mathrm{m}^{2}$ foram caracterizados como eutróficos, valores de 25,0 a $29,9 \mathrm{Kg} / \mathrm{m}^{2}$ 
como sobrepesados e de $30,0 \mathrm{Kg} / \mathrm{m}^{2}$ ou superior, como obesos.

Tabela 1. Classificação do Índice de Massa Corporal segundo OMS.

\begin{tabular}{cc}
\hline IMC & Classificação \\
\hline$<18,5$ & Abaixo do Peso \\
$18,6-24,9$ & Saudável \\
$25,0-29,9$ & Peso em Excesso \\
$30,0-34,9$ & Obesidade Grau I \\
$35,0-39,9$ & Obesidade Grau II (severa) \\
$\geq 40,0$ & Obesidade Grau III (mórbida) \\
\hline Fonte: WHO-World Health Organization ${ }^{10}$
\end{tabular}

Para a determinação da \%GC, foi utilizada a classificação de McCardle et al., $(1998)^{11}$, onde valores de $25-30 \%$ são correspondentes a Eutrofia, de $30-35 \%$ a Sobrepeso e $35-40 \%$ a Obesidade.

A obesidade abdominal (OAB) foi determinada a partir do valor maior que $80 \mathrm{~cm}$ para mulheres e $94 \mathrm{~cm}$ para homens, obtido a partir da mensuração da Circunferência Abdominal (CAb). Dessa maneira, indivíduos que apresentavam valores superiores, foram classificados como obesos. Dentro dessa categoria, foi estabelecido grau I (homens: 94$101 \mathrm{~cm}$; mulheres: $80-87 \mathrm{~cm})$ ou grau II (homens: $\geq 102 \mathrm{~cm}$; mulheres: $\geq 88 \mathrm{~cm}$ ). Em contrapartida, participantes com valores inferiores foram classificados como eutróficos, ou ausência de obesidade.

Análise da prevalência de doenças

Para a determinação da ocorrência de doenças, foi utilizada uma anamnese baseada no Standard Health Questionnaire (SHQ) validado pelo "Washington State Health Insurance Pool”12. Tal instrumento aborda a presença ou ausência da enfermidade no indivíduo avaliado em um período de até 10 anos. Nesse sentido, foram agrupadas de acordo com a sua ocorrência, em três categorias: Doenças Cardíacas, Doenças Metabólicas /Endócrinas, Doenças Musculares /Ortopédicas.

Análise estatística e ilustração dos resultados

Foi determinado o ponto de corte de acordo com a variável idade (i) e utilizada a análise de frequência para a distribuição das variáveis da composição corporal com o inquérito de doenças (ii). Por último, foi aplicado o teste do Qui-Quadrado, para análise da associação entre variáveis (iii). Para isso, utilizou-se o aplicativo estatístico SPSS $10.0^{\circledR}$, adotando-se um erro de $5 \%$ (valor de $p=0,05)$. A elaboração dos gráficos foi realizada por meio do Software "Origin" versão $6.0^{\circledR}$.

\section{RESULTADOS}

Foi possível observar valores médios correspondentes a eutrofia a partir do IMC $(24,7)$. No entanto, os valores de $O A B$ mostram-se elevados, uma vez que o contingente amostral é composto também por mulheres, o que o torna acima dos padrões recomendados para indivíduos deste 
sexo $(80 \mathrm{~cm})$. Tais achados podem ser melhor

observados logo abaixo (Tabela 2).

Tabela 2. Características antropométricas da amostra analisada [n=77].

\begin{tabular}{ccccc}
\hline & \multicolumn{4}{c}{ Caracterização Amostral } \\
\hline Idade & Média & Desvio Padrão & Mínimo & Máximo \\
Peso & 29,99 & 9,36 & 18 & 59 \\
Altura (m) & 72,33 & 14,43 & 47,4 & 117 \\
IMC & 1,69 & 0,08 & 1,53 & 1,89 \\
\%GC & 24,7 & 4,8 & 15,9 & 36,9 \\
CAb & 24,6 & 8,37 & 7,9 & 41,1 \\
\hline
\end{tabular}

Nota: (\%GC) Percentual de gordura corporal. CAb: Circunferência Abdominal. IMC: Índice de Massa Corporal.

No presente estudo, é possível notar maiores indicadores da composição corporal no grupo de indivíduos com idade superior a 30 anos, como o peso corporal, IMC, \%GC e
OAB. Nesse sentido, observa-se o aumento da adiposidade em decorrência da idade (Gráfico 1).

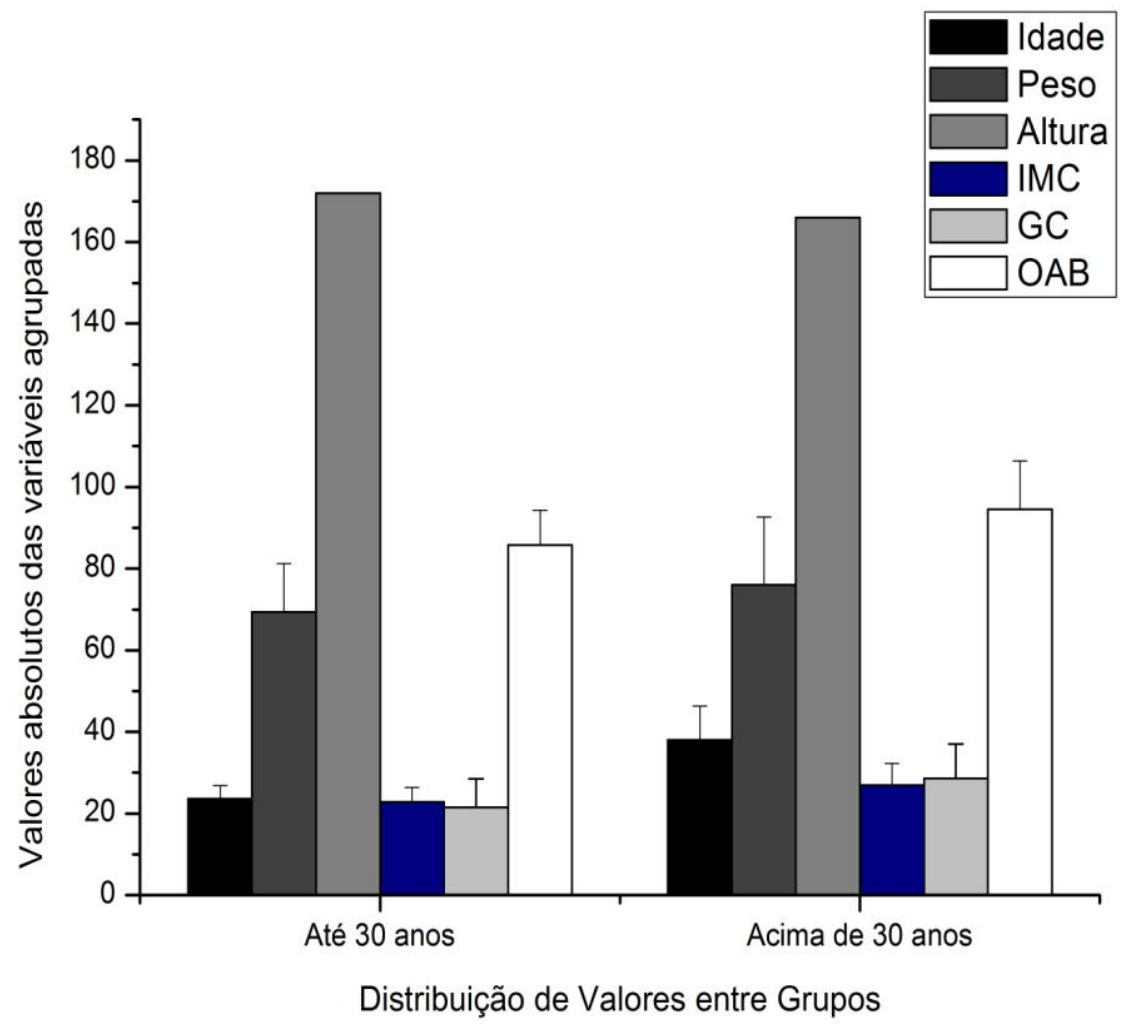

Gráfico 1. Caracterização entre os dois grupos analisados. (\%GC) Percentual de gordura corporal. CAb: Circunferência Abdominal. IMC: Índice de Massa Corporal. 
Nota-se que, quando tomado o IMC como parâmetro para a análise de sobrepeso e obesidade, o grupo de indivíduos com idade inferior a 30 anos, apresenta-se em sua maioria em estado de eutrofia (69,8\%). Já o grupo com idade superior, apresenta $44,1 \%$ de indivíduos eutróficos, enquanto $32,4 \%$ são sobrepesados. Nesse último caso, observa-se que $23,5 \%$ são obesos, fato que não foi observado no primeiro grupo. Em relação ao \%GC, quando se observa o grupo de indivíduos com faixa etária superior, nota-se que $21,9 \%$ desses indivíduos apresentam-se obesos mórbidos. Tal achado não ocorreu no grupo de indivíduos com menor idade (Tabela 3).

O presente estudo observou a prevalência de obesidade abdominal nos diferentes grupos etários. No grupo inferior a 30 anos de idade foi observada a ausência em $60,5 \%$ dos avaliados. No entanto, no grupo com idade superior, a presença dessa patologia foi bem mais acentuada, nesse caso,
76,5\% dos participantes apresentaram obesidade na região abdominal graus I ou II (Tabela 3).

O presente estudo também se propôs a observar a ocorrência de doenças cardíacas. Nota-se, ligeiro aumento nos indivíduos com idade superior a 30 anos. Neste caso, a ocorrência foi 7,4\% superior a indivíduos com menor idade. Tal fato se repete quando foi observada a prevalência de metabólicas endócrinas. Nesse caso, houve um ligeiro aumento nos casos de doenças, nos indivíduos com idade superior a 30 anos (2,2\%). Na análise da prevalência de doenças musculares ortopédicas, foi observado maior ocorrência em indivíduos com idade superior a 30 anos. Neste caso, foi possível notar a presença dessa patologia 64,33\% superior $(16,3$ contra $29,4 \%)$ ao grupo com menor idade (Tabela 3). 
Tabela 3. Associação da composição corporal e ocorrência de doenças crônico-degenerativas em indivíduos com idade inferior e superior a 30 anos [ $\mathrm{N}=77]$.

Índice de Massa Corporal

\begin{tabular}{cccccc}
\hline Idade & Eutrofia & Sobrepeso & Obesidade & Total & $\mathrm{X}^{2}$ \\
Até 30 anos & 69,8 & 27,9 & 2,3 & 100 & 0,01 \\
$>30$ anos & 44,1 & 32,4 & 23,5 & 100 & 0,33
\end{tabular}

\section{Gordura Corporal}

\begin{tabular}{|c|c|c|c|c|c|c|c|}
\hline Idade & Eutrofico & Sobrepeso & $\begin{array}{l}\text { Obeso } \\
\text { Grau I }\end{array}$ & $\begin{array}{l}\text { Obeso } \\
\text { Grau II }\end{array}$ & $\begin{array}{l}\text { Obeso } \\
\text { Grau III }\end{array}$ & Total & $x^{2}$ \\
\hline Até 30 anos & 31,0 & 40,5 & 23,8 & 4,8 & --- & 100 & 0,01 \\
\hline$>30$ anos & 18,8 & 12,5 & 28,1 & 18,8 & 21,9 & 100 & 0,72 \\
\hline \multicolumn{8}{|c|}{ Obesidade Abdominal } \\
\hline \multicolumn{2}{|l|}{ Idade } & Ausência & Grau 1 & Grau 2 & Total & \multicolumn{2}{|r|}{$\mathrm{X}^{2}$} \\
\hline \multicolumn{2}{|c|}{ Até 30 anos } & 0,5 & 25,5 & 14,0 & 100 & \multicolumn{2}{|c|}{0,01} \\
\hline \multicolumn{2}{|l|}{$>30$ anos } & 3,5 & 23,5 & 53,0 & 100 & \multicolumn{2}{|c|}{0,05} \\
\hline
\end{tabular}

\section{Doenças Cardíacas}

\begin{tabular}{ccccc}
\hline Idade & Ausência & Presença & Total & $X^{2}$ \\
Até 30 anos & 72,1 & 27,9 & 100 & 0,04 \\
$>30$ anos & 64,7 & 35,3 & 100 & 0,08 \\
\hline \multicolumn{2}{l}{ Doenças Metabólicas Endócrinas } & & & $X^{2}$ \\
\hline Idade & Ausência & Presença & Total & 0,01 \\
$>30$ anos & 69,8 & 30,2 & 100 & 0,04
\end{tabular}

\section{Doenças Musculares Ortopédicas}

\begin{tabular}{ccccc}
\hline Idade & Ausência & Presença & Total & $X^{2}$ \\
Até 30 anos & 83,7 & 16,3 & 100 & 0,01 \\
$>30$ anos & 70,6 & 29,4 & 100 & 0,01
\end{tabular}

Nota: $X^{2}$ : Teste do Qui-Quadrado para a análise da associação entre variáveis com erro de $5 \%(p=0,05)$. Valores percentuais em cada uma das categorias (\%). 


\section{DISCUSSÃO}

Este modelo de estudo buscou evidenciar a associação da prevalência de sobrepeso e obesidade, além da ocorrência de DCD, em indivíduos com diferentes faixas etárias, de ambos os sexos. Os resultados apresentados foram obtidos a partir de amostra de 77 indivíduos da cidade de Presidente Prudente/SP, ingressantes em um programa de atividade física, oferecido por uma academia de musculação deste município.

No presente estudo, foi possível observar que indivíduos com idade até 30 anos (grupo1), apresentam prevalência de eutrofia, a partir dos valores de IMC, \%GC e $\mathrm{OAB}$. Porém, estes valores se invertem no decorrer da idade, havendo prevalência de sobrepeso e obesidade acima dos 30 anos. Além disso, os valores percentuais mostram maior ocorrência de obesidade graus I, II e III em indivíduos com idade mais avançada.

Tal fato sugere que a partir dos 30 anos de idade, os indivíduos tornam-se mais suscetíveis a apresentar sobrepeso e obesidade. Dessa forma, o estilo de vida, cuidados com a alimentação e a prática de atividade física devem ser revistos. No Brasil, estudos $^{13}$ observaram o aumento da prevalência de obesidade em adultos, com proporções de $2,8 \%$ e $12,4 \%$ em homens no período de 1974 e 2009, respectivamente, e de $8,0 \%$ e $16,9 \%$ nas mulheres no mesmo período.

Os indicativos desta pesquisa demonstra certa preocupação com a população analisada, pois maior adiposidade aumenta o risco de desenvolver doenças como Hipertensão Arterial Sistêmica, Diabetes Tipo II, Obesidade e Síndrome Metabólica, além de reduzir a expectativa e qualidade de vida $^{14,4,15}$. Outro ponto a ser destacado foi o aumento circunferencial abdominal. Enquanto o grupo de indivíduos com idade inferior a 30 anos possuía $14 \%$ dos casos de obesidade abdominal Grau II, 53,0\% dos indivíduos com idade superior se enquadravam nesta categoria. $\mathrm{O}$ acúmulo de gordura nesta região é um fator pré-disposicional ao surgimento de patologias como arteriosclerose, diabetes e doenças cardíacas $^{16,17}$. Alguns autores ${ }^{15}$ observaram que o exercício físico aeróbio se mostrou eficiente na diminuição do tecido adiposo em indivíduos sobrepesados e obesos.

Por abordar patologias, o presente estudo observou associação favorável ao surgimento de diversas doenças em indivíduos com idade superior a 30 anos. Já é sabido que indivíduos que não possuem hábitos de saúde considerados adequados tendem a desenvolver DCD. Tais males surgem após certo período de tempo, e nesse caso, idosos tendem a apresentar estas doenças que foram desencadeadas ao longo 
da vida, principalmente quando não participam regularmente de um programa de exercícios físicos ${ }^{4}$. Segundo alguns estudos, a prevalência HAS detém-se em torno de $50 \%$ a $70 \%$, respectivamente, a partir dos 60 e 70 anos de idade $^{18}$. Neste sentido, a HAS tem uma alta prevalência entre adultos, tendendo a afetar em maior número mulheres do que homens em todo o mundo ${ }^{19}$.

Nos casos de doenças metabólicas endócrinas, observou-se um ligeiro aumento no grupo de indivíduos com idade superior a 30 anos. Dentre as principais doenças categorizadas nesse grupo, a mais comumente presente é o Diabetes Mellitus Tipo II, que é caracterizado pela ocorrência de hiperglicemia. Alguns autores ${ }^{20}$ afirmam que esta patologia tem emergido e se tornou um dos principais alarmes para a saúde humana no século XXI. Que são necessárias mudanças de comportamento e no estilo de vida da população. Uma das formas de combatê-la é a adesão a programas de exercício físico. Estudos mostram que o exercício físico pode ser um auxiliar no combate e prevenção ao Diabetes Mellitus II por interagir no sistema metabólico (aumento da sensibilidade) e diminuir a taxa de glicose sanguínea ${ }^{20,21}$.

Quando verificados os casos de doenças musculares ortopédicas, observou-se uma maior ocorrência de doenças em indivíduos com maior idade. A osteoporose é o maior problema de saúde púbica mundial e está associado significativamente a problemas de morbidade, mortalidade e de gastos econômicos ${ }^{22}$.

Além disso, a Sociedade Científica Internacional de Reabilitação e Tratamento de Escoliose Ortopédica ${ }^{23}$, recomenda o tratamento de doenças como escoliose, que podem ser tratadas por meio de fisioterapia, órteses e exercícios específicos. Nesse sentido, a aderência a um programa de exercícios físicos, torna-se fundamental para o combate a estes males.

Sendo assim, o presente estudo contribui com a literatura ao identificar a prevalência de sobrepeso e obesidade em indivíduos de diferentes faixas etárias. Além de verificar a associação entre $D C D$ com o excesso de peso corporal. A presente pesquisa pode ser utilizada por autoridades governamentais com a intenção de aderir a políticas públicas com a finalidade da promoção à prática de atividade física e redução do peso corporal.

Porém, há limitações quanto à população e local utilizado para coleta de dados. Estudos de outras localidades podem vir a complementar a presente pesquisa, podendo assim, serem comparados os valores de diferentes regiões do município ou de outras cidades brasileiras. Deste modo, sugere-se a realização de pesquisas desta natureza em diferentes centros urbanos, a fim de observar as características da população, 
que é marcantemente diferenciada por fatores ambientais, comportamentais, sociais, entre outros.

\section{CONCLUSÃO}

Conclui-se que indivíduos com maior idade, possuem maior prevalência de sobrepeso e obesidade, assim como uma maior suscetibilidade a ocorrência de doenças crônico-degenerativas.

\section{REFERÊNCIAS}

1. Organização Pan-Americana de Saúde; Organização Mundial de Saúde. Doenças crônico-degenerativas e obesidade: estratégia mundial sobre alimentação saudável, atividade física e saúde. Brasília: Ministério da Saúde; Organização PanAmericana de Saúde; Organização Mundial de Saúde; 2004.

2. WHO - World Health Organization. World health statistics. Geneva: WHO; 2009.

3. Guh DP, Zhang W, Bansback, Amarsi $Z$, Birmingham $C L$, Anis $H A$. The incidence of co-morbidities related to obesity and overweight: a systematic review and metaanalysis. BMC Public Health 2009; 9:88. DOI: http://dx.doi.org/10.1186/1471-2458-9-88

4. Castoldi RC, Moret DG, Gomes IC, Paulo TRS, Oikawa SM, Freitas Júnior IF. Influência da adiposidade corporal sobre a aptidão cardiorrespiratória em mulheres idosas. $\mathrm{R}$ Bras Ci e Mov. 2010; 18(4):34-38.

5. Lima MT, Bucher JSNF, Lima JWO. A hipertensão arterial sob o olhar de uma população carente: estudo exploratório a partir dos conhecimentos, atitudes e práticas. Cad Saúde Pública 2004; 20(4):1079-1087.
DOI: $\quad$ http://dx.doi.org/10.1590/S0102311X2004000400023

6. Selby JV, Friedman GD, Quesenberry CP Jr. Precursors of essential hypertension. The role of body fat distribution pattern. Am J Epidemiol. 1989; 129:43-53.

7. Hans TS, Van Leer EM, Seidell JC, Lean MEJ. Waist circumference action levels in the identification of cardiovascular risk factors: prevalence study in a random sample. BMJ 1995; 311:1401-1405.

8. Carneiro G, Faria AN, Ribeiro Filho FF, Guimarães A, Lerário $D$, Ferreira SRG et al. Influência da distribuição da gordura corporal sobre a prevalência de hipertensão arterial e outros fatores de risco cardiovascular em indivíduos obesos. Rev Assoc Med Bras. 2003; 49(3):306-311. DOI: http://dx.doi.org/10.1590/S010442302003000300036

9. Santarém JM. Musculação e qualidade de vida. Rev Bras Med Esporte. 1996; 2: 11-4.

10. WHO - World Health Organization. World health statistics. Geneva: WHO; 2008.

11. McArdle WD, Katch F I, Katch VL. Fisiologia do exercício: energia, nutrição e desempenho Humano. 4. ed. Rio Janeiro: Guanabara Koogan; 1998.

12. WSHIP - Washington State Health Insurance Pool. Standard Health Questionnaire. Washington: WSHIP; 2009.

13. Sousa TF, Nahas MV, Silva DAS, Duca GFD, Peres MA. Fatores associados à obesidade central em adultos de Florianópolis, Santa Catarina: estudo de base populacional. Rev Bras Epidemiol. 2011; 14(2):296-309. DOI: http://dx.doi.org/10.1590/S1415790X2011000200011 
14. Koning L, Merchant AT, Pogue J, Anand SS. Waist circumference and waist-to-hip ratio as predictors of cardiovascular events: meta-regression analysis of prospective studies. Eur Heart J. 2007; 28(7):850-6.

15. González Calvo G, Hernández Sánchez S, Pozo Rosado P, García López D. Positive effects of physical exercise on reducing the relationship between subcutaneous abdominal fat and morbility risk. Nutr Hosp. 2011; 26(4):685-91.

16. Rezende FAC, Rosado LEFPL, Ribeiro RCL, Vidigal FC, Vasques ACJ, Bonard IS et al. Índice de massa corporal e circunferência abdominal: associação com fatores de risco cardiovascular. Arq Bras Cardiol. 2006; 87(6): 728-73.

DOI:

http://dx.doi.org/10.1590/S0066-

782X2006001900008

17. Pitanga F. Anthropometry for the assessment of abdominal obesity and coronary risk. Rev Bras Cineantropom Desempenho Hum. 2011; 13(3):238-241. DOI: http://dx.doi.org/10.5007/1980-

0037.2011v13n3p238

18. Rosa EC, Plavnik FL, Tavares A. Hipertensão arterial sistêmica. Rev Bras Med. 2006; 63(1/2):5-17.

19. Krause MP, Hallage T, Gama MPR, Miculis CP, Matuda NS, Silva SG. Associação de aptidão cardiorrespiratória e circunferência abdominal com hipertensão em mulheres idosas brasileiras. Arq Bras Cardiol. 2009; 93(1):2-8.

DOI:

http://dx.doi.org/10.1590/S0066-

782X2009000700002

20. Teixeira-Lemos E, Nunes S, Teixeira F, Reis F. Regular physical exercise training assists in preventing type 2 diabetes devolopment: focus on its antioxidant and anti-inflamatory properties. Cardiovasc Diabetol. 2011;10-12.
DOI: http://dx.doi.org/10.1186/1475-284010-12

21. Colberg SR, Sigal RJ, Fernhall B, Regensteiner JG, Blissmer BJ, Rubin RR et al. Exercise and type 2 diabetes. Diabetes Care 2010; 33(12):147-167. DOI: http://dx.doi.org/10.2337/DC10-9990

22. Aggarwal N, Raveendran A, Khandelwal $\mathrm{N}$, Sen RK, Thakur JS, Dhaliwal LK, et al. Prevalence and related risk factors of osteoporosis in peri- and postmenopausal Indian women. J Midlife Health. 2011; 2(2): 81-85. DOI: http://dx.doi.org/10.4103/09767800.92537

23. Negrini $S$, Aulisa AG, Aulisa L, Circo AB, Mauroy JC, Durmala J et al. 2011 SOSORT guidelines: orthopaedic and rehabilitation treatment of idiopathic scoliosis during growth. Scoliosis 2012; 7:3. DOI: http://dx.doi.org/10.1186/1748-7161-7-3

Recebido para publicação em 18/08/2014 Revisado em 08/09/2014

Aceito em 12/09/2014 